\title{
Vom Beruf zur Profession
}

\author{
Die »Qualitätsziele der Wohlfahrtsverbände« im Lichte der \\ Profession Sozialer Arbeit
}

Silvia Staub-Bernasconi

In den Blättern der Wohlfahrtspflege 3/2009 hat die Bundesarbeitsgemeinschaft der Freien Wohlfahrtspflege e.V. ihre Qualitätsziele im Hinblick auf Hilfeleistungen konkretisiert und praxisorientiert anhand einer Übersicht formuliert (S. 85-88). Damit "sind sie Umsetzungshilfe und Prüfungsgrundlage für die Selbstbewertung durch MitarbeiterInnen und Fremdbewertung mittels Zertifizierung « (S. 85). Ich habe a.a.O. dargelegt, dass sich die Soziale Arbeit an einem Scheideweg befindet: Wird oder bleibt sie ein weisungsgebundener Beruf mit einem Doppelmandat seitens der Träger und der Klientel oder ist bzw. wird sie zur Profession mit einem Tripelmandat, wobei das dritte Mandat von der Profession ausgeht und durch Wissenschaftsbasierung ihrer Handlungsvollzüge sowie einen Ethikkodex zu charakterisieren ist? (Staub-Bernasconi 2010) Aufgrund internationaler Standards gründet dieser Kodex seine Ethik auf die Menschenrechte und soziale Gerechtigkeit (vgl. Supplement Journal of International Social Work, 2007). Dieses dritte Mandat verleiht ihr eine relative Autonomie und kritische Urteilskraft gegenüber Ansprüchen sowohl seitens der Arbeitgeber als auch seitens der AdressatInnen Sozialer Arbeit.

Prof. Dr. habil. Silvia Staub-Bernasconi war Dozentin für Soziale Arbeit von 1967 bis 1996 an der Hochschule für Soziale Arbeit Zürich und von 1996 bis 2003 an der Technischen Universität Berlin. Von 2002 bis 2010 leitete sie den Masterstudiengang "Soziale Arbeit als Menschenrechtsprofession « in Berlin. Seit 2007 ist sie Mitglied des Academic Board und Lehrbeauftragte von INDOSOW - International Doctoral Studies of Social Work (Internet http://www.indosow.net).

E-Mail staubernasco@bluewin.ch

\section{Das Dokument »Qualitätsziele der Wohlfahrtsverbände zur Erreichung ihrer spezifischen Dienstleistungsqualität «}

Diese Ziele werden anhand von acht Teilanforderungen - spezifiziert als »Grundanliegen «, »Qualitätsanforderungen « und »Prüfkriterien « - operationalisiert. Ich kommentiere hier diejenigen Aussagen, die ich aus der Perspektive der erwähnten Weichenstellung - Beruf oder Profession - als entscheidend betrachte:

\section{Erstes Grundanliegen: \\ Leitbildorientierung}

Leitbilder stellen einen wertebezogenen Orientierungsrahmen dar, die als verpflichtende Grundlage für das berufliche und organisatorische Handeln dienen. ... (Sie) ermöglichen, ethische Fragen bei konkurrierenden Interessen zu klären. ... (Es) enthält Aussagen über Aufgaben, Zweck, Menschenbild, Werteorientierung. ... Die Qualitätspolitik und/oder strategie der Organisation wird aus dem Leitbild abgeleitet:

(Die in Anführungszeichen gesetzten Sätze und Satzteile sind Originalzitate aus dem Grundsatzpapier.)

Dabei wird festgehalten, dass zum einen das Leitbild partizipativ entwickelt wird und zum andern, dass es den MitarbeiterInnen vermittelt... und den NutzerInnen bekanntgemacht wird. Es ist aufgrund des Textes nicht erkennbar, ob an diesem Leitbild auch SozialarbeiterInnen mitgewirkt haben bzw. wer ein Anrecht auf Mitbestimmung bei dessen Weiterentwicklung hat.

\section{Zweites Grundanliegen: Orientierung am persönlichen Nutzen}

Der Untertitel des Heftes »Vom Klienten zum Kunden « ist bereits ein Hinweis auf das gewählte Menschenbild. Diese soziale Kategorie wird in der Übersicht spezifiziert: NutzerInnen, PatientInnen, KlientInnen oder ratsuchende Menschen betrachten wir unter Achtung ihrer Souveränität, Selbstbestimmung und Kreativität als KundInnen. Diese Orientierung ist eine Abkehr von einem institutionsorientierten Denken zu einem personorientierten Handeln, ... bei dem die Lebensqualität und das Wohlbefinden der NutzerInnen als Messgrößen der Qualität von Leistungen in den Fokus genommen wird. ... . Das heißt, dass die Organisation die NutzerInnen über das Dienstleistungsangebot und die Dienstleistungsgestaltung (informiert) ... wobei die Erwartungen und Wünsche der NutzerInnen dokumentiert (sind).

Wie kommt es, dass Wohlfahrtsverbände mit mehrheitlich konfessionellem Anteil, die gewiss auf andere, "menschlichere« Menschenbilder zurückgreifen könnten, sich ausgerechnet dem Bild eines Menschen verschreiben und unterordnen, der - ohne sozialen Bezug auf Mitmenschen - offenbar nichts anderes im Sinn hat, als auf einem Dienstleistungsmarkt seinen individuellen Nutzen in Form von Wünschen zu verfolgen? So wird hier »Qualität « mit individueller Nutzenmaximierung gleichgesetzt.

Wie soll man aber eine menschliche Hilfsbeziehung nutzen, benutzen, vernutzen oder gar verbrauchen? Dass dieser Mensch als erstes ein Hilfe benötigender Mensch in Not sein könnte, ist nicht angedacht. Wenn er nämlich, wie vorausgesetzt, souverän, selbstbestimmt und kreativ wäre, hätte er auch keinen Hilfebedarf. Diese Eigenschaften individueller »Fitness « werden normativ, d. h. der Klientel Sozialer Arbeit ohne Bezug auf empirisch erhobene, reale Lebenssituationen und Merkmale per Definition zugeschrieben. KundInnen sind Menschen, die sich auf einem Markt mit eigenen finanziellen Ressourcen und halbwegs frei wählbarem Angebot bewegen, Produkte, die ihnen nicht passen, ablehnen, zurükkgeben oder umtauschen können. Inwiefern sind da Jugendliche ohne Schulabschluss und Lehrstelle, Menschen ohne ei- 
genes Einkommen, ferner von der Sozialhilfe abhängige Familien, Langzeitarbeitslose, unbegleitete Minderjährige, Straffällige, politisch Verfolgte, Asylsuchende ohne Papiere, Rassismusopfer, Vergewaltiger, Opfer von Frauen- und Kinderhandel u. v. m. gemeint? Oder geht es eventuell implizit um mittelständische AdressatInnen, die sich dank ihrer finanziellen Ressourcen als zahlende KundInnen souverän auf einem Kultur- und Freizeitmarkt, einem Altersheim- und Pflegeheimmarkt bewegen können? Ist da nicht bezüglich der »Verbraucher der Sozialen Arbeit « etwas an Realitätsbezug aus den Fugen geraten? Die systematische Eliminierung der Thematisierung des millionenfachen Leidens von Menschen, ihrer Unrechtserfahrungen - lokal, national und weltweit - bis in die Fachsprache der Sozialen Arbeit ist eines der größten Erfolge der weltweiten, neoliberalen Semantik und Ideologie (Meyer 2005).

Dieses verstümmelte Menschenbild der neoklassischen Ökonomie ist wissenschaftlich unhaltbar und wird nicht einmal mehr von Ökonomen aufrechterhalten (vgl. die letzten Nobelpreise in Ökonomie, ferner Gintis et al., 2005, Frey 2004, Frey/Frei-Marti 2010). Auch im medizinisch-psychiatrischen Bereich rekurriert man auf ein mehrdimensionales biopsychosoziales - Menschenbild, das in der Sozialen Arbeit noch um die kulturelle Dimension zu erweitern wäre. Dazu käme die Vorstellung der grundsätzlichen Abhängigkeit des Menschen von anderen Menschen und den von ihnen geschaffenen sozialen Systemen für seine Bedürfnisbefriedigung und Wunscherfüllung. Und diese Abhängigkeit ist es, die auch seine Verletzbarkeit ausmacht. Darum spricht man im Menschenrechtsdiskurs von »vulnerable groups «. Sie stimmen fast durchgängig mit den in den Spezialkonventionen erwähnten AdressatInnen (Kinder, Frauen, MigrantInnen, politische Flüchtlinge usw.) überein.

Menschenrechte und die Vorstellung von unverlierbarer Menschenwürde sind in diesem Zusammenhang ein klares Korrektiv für das Schrumpfmodell des homo oeconomicus der neoklassischen Mainstream-Ökonomie. Überdies ist die Soziale Arbeit nicht verpflichtet, Wünsche zu erfüllen - diese sind grenzenlos und kulturell codiert. Zudem gibt es legitime und illegitime Wünsche; letztere sind solche, welche die Bedürfniserfüllung anderer
Menschen be- oder verhindern. Hingegen ist Soziale Arbeit gemäß ihrem nationalen und internationalen Ethikkodex verpflichtet, die Bedürfnisbefriedigung ihrer AdressatInnen und damit ihr Wohlbefinden zu ermöglichen, falls sie dies nicht aufgrund eigener, individueller Ressourcen und des diskriminierungsfreien Zugangs zum Bildungs-, Wirtschafts-, kulturellen System tun können, weil sie sozialstrukturellen Unrechtserfahrungen unterworfen sind. Soziale Ar- beit hat aber auch den Auftrag, im und mit dem problemverursachenden, z. B. diskriminierenden, ausbeuterischen, repressiven, gewaltfördernden sozialen und kulturellen Umfeld zu arbeiten. Sie kann deshalb nicht nur personorientierte Dienstleistung sein.

\section{Grundanliegen: Orientierung an Gemeinwesen und Gesellschaft \\ Unter dieser Leitlinie sollen das $\mathrm{Ge}$ - meinwesen gestärkt, Partizipation orga-}

\section{Literatur}

Bielefeldt, Heiner (2004): Die Würde als Maßstab, In: Mahler, C./Mihr, A. (Hg.): Menschenrechtsbildung. Bilanz und Perspektiven, VS Verlag für Sozialwissenschaften, Wiesbaden, S. 19-27.

Exworthy Mark/Halford Susan (Eds.) (1999): Professionals and the New Managerialism in the Public Sector, Buckingham/Philadelphia.

Frey, Bruno S (2008): Happiness: A Revolution in Economics, MIT Press, Cambridge, MA and London, UK, 2008, 240 p.

Frey, Bruno S./Frei-Marti, Claudia (2010): Glück. Die Sicht der Ökonomie, Rüegger, Zürich.

Galuske, Michael/Thole, Werner (2006): Vom Fall zum Management. Neue Methoden der Sozialen Arbeit, VS Verlag für Sozialwissenschaften, Wiesbaden.

Gintis, Herbert/Bowles, Samuel/Boyd, Robert T./Fehr, Ernst (Eds.) (2005): Moral Sentiments and Material Interests : The Foundations of Cooperation in Economic Life (Economic Learning and Social Evolution), MIT Press, Cambridge.

Gore, Madhov S. (1969): Social Work and ist Human Rights Aspcts, in: Proceedings of the XIVth Int. Conference on Social Welfare, Helsinki, S. 56-68.

Hansen, Eckhard (2006): Das Case/Care Management, In: Galuske, Michael/Thole, Werner (Hg.): Vom Fall zum Management. VS Verlag für Sozialwissenschaften, Wiesbaden, S. 17-36.

Meyer, John W. (2005): Weltkultur. Wie die westlichen Prinzipien die Welt durchdringen. Suhrkamp, Frankfurt am Main.

Supplement des Journal International Social Work, Sage, London (2007): International Definition of the Social Work Profession - Ethics in Social Work - Global Standards for the Education and Training of the Social Work Profession, English Version, p. 3-41.

Staub-Bernasconi, Silvia (2007) Soziale Arbeit: Dienstleistung oder Menschenrechtsprofession? Zum Selbstverständnis Sozialer Arbeit in Deutschland mit einem Seitenblick auf die internationale Diskussionslandschaft, In: Lob-Hüdepohl, Andreas/Lesch, Walter (Hg.): Einführung in die Ethik der Sozialen Arbeit, UTB/Schöningh, Paderborn, 2007: S. 20-53.

Staub-Bernasconi, Silvia (2008): Soziale Arbeit und Menschenrechte - Oder: Was haben Menschenrechte in der Sozialen Arbeit zu suchen?, In: Widersprüche, Kleine, Bielefeld, Jan. 2008, H. 107, S. 9-32.

Staub-Bernasconi, Silvia (2009): Der Professionalisierungsdiskurs zur Sozialen Arbeit (SA/SP) im deutschsprachigen Kontext im Spiegel internationaler Ausbildungsstandards, In: Becker-Lenz, Roland et al.: Professionalität in der Sozialen Arbeit, VS Verlag für Sozialwissenschaften, Wiesbaden, S. 21-45.

Staub-Bernasconi, Silvia (2010): Soziale Arbeit als Handlungswissenschaft, UTB/Haupt, Bern/Stuttgart/Wien, 2. Aufl.

Thornhill, Christ/Ashenden, Samantha (eds.) (2010): Legality and Legitimacy: Normative and Sociological Approaches, Nomos, Baden-Baden. 
nisiert, Bürgerbeteiligung gefördert und Lebenslagen verbessert sowie Integration und Solidarität vertieft (werden). Die Mittel dazu sind, wenn ich richtig sehe, ehrenamtliches Engagement, das die Qualität Sozialer Arbeit bereichern soll, ferner Zusammenarbeit (der Träger) im Gemeinwesen/Sozialraum, wodurch Synergieeffekte entstehen sollen.

In der Operationalisierung dieser an sich zu bejahenden Zielvorstellungen zeigt sich, dass die Marschrichtung vornehmlich aus der Perspektive der Organisationen vorgegeben wird: So ist die Verankerung der Organisation im Gemeinwesen Führungsaufgabe und nicht etwa die klassische Aufgabe von Sozialarbeitenden, in demokratisch-partizipativer Zusammenarbeit mit den Einwohnern von Nachbarschaften, Stadtteilen, den Mitgliedern von Grassrootorganisationen, NGOs, mit LokalpolitikerInnen und den Trägerinstanzen. Ferner wird davon ausgegangen, dass die Organisation Informationen über mögliche Aufgaben und Einsatzfelder freiwillig Engagierten im Sozialraum zur Verfügung stellt. Nirgends ist festgehalten, dass sich StadtteilbewohnerInnen zusammentun, um - befähigt und unterstützt durch professionelle SozialarbeiterInnen - die Beschaffung von Informationen, die Formulierung von Bedürfnissen, Interessen, Zielen und die Verteilung von Aufgaben zur Problemlösung zu übernehmen. Nirgends ist vorgesehen, dass EinwohnerInnen ihre Rechte, mit eingeschlossen Menschenbzw. Sozialrechte einfordern, je nachdem Missstände und Willkür, illegitime Machtausübung und Verfahrenswillkür thematisieren können und dabei die Chance haben seitens der Träger auf offene Ohren zu stoßen.

\section{Grundanliegen: Ziel- und \\ Wirkungsorientierung}

Diese Leitlinie zwinge dazu, klare Ziele zu definieren und Erfolgs-/Ergebniskriterien zu beschreiben. ... Der Regelkreislauf aus Zielformulierung, Maßnahmenplanung, Umsetzung, Evaluation/Messung der Ergebnisse und erneuter Zielformulierung ist als systematisches Verfahren dokumentiert und in der Praxis nachweisbar.

Der als Prüfkriterium definierte »Regelkreislauf « verweist am klarsten auf die Weichenstellung zwischen dem eingangs beschriebenen Berufs- versus Professionsszenario. Er beginnt mit der "Zielformulierung ", so dass vermutet werden muss, dass auf eine Beschreibung und Erklärung der Problemsituation - beides unhintergehbare Anforderungen an eine Profession - verzichtet werden kann, weil es um die Zuordnung zwischen zumeist standardisierten (u. a. rechtlichen) »Maßnahmen« und »Wünschen « geht. (Ein Verkäufer fragt eine Käuferin auch nicht nach ihrer Lebenssituation, ihren tieferen Motiven für ihren Kauf!) Mit dieser stark verkürzten methodischen Fallbearbeitung und dem Doppelmandat, das zwischen Träger und Nutzer vermittelt, wird Soziale Arbeit programmatisch auf dem Berufsniveau festgehalten und dort, wo sie sich zur Profession entwickelt hat oder entwickeln will, auf kaltem Wege entprofessionalisiert.

Eine Menschenrechtsorientierung als allgemeine regulative Idee Sozialer Arbeit wird darüber hinaus die diagnostische Frage nach geringfügigen, mittleren, schwerwiegenden Menschenrechtsverletzungen stellen. Besonders schwierig gestaltet sich die Antwort auf die Frage nach Täter- und Opferverhalten, wenn beides bei der gleichen Person der Fall sein kann. Dabei ist zu beachten, dass Menschenrechte nicht zur allzeit bereiten Moralkeule werden dürfen.

\section{Grundanliegen: Vertragspartnerschaft}

Die Woblfahrtsverbände gehen davon aus, dass der Dienstleistungsvertrag mit den NutzerInnen ... diesen Rechtssicherheit (gibt) (Stichwort »Rechtskonforme Vertragsklauseln"). ... Vor Vertragsabschluss werden die Vertragspartner umfassend über die Vertragsinhalte informiert. ... Über absehbare Veränderungen werden die Vertragspartner frühzeitig informiert. Die Verträge enthalten Vertragsdauer, Leistungsart, Leistungsumfang, Häufigkeit und Dauer der Leistung, Einzelpreise und Gesamtkosten, Name der Leitung, unabhängige Beschwerdestelle, Kündigungsmodalitäten.

Einseitige Informationsabgabe kann wohl kaum als »Partnerschaft « im Rahmen einer »koproduktiven Arbeitsbeziehung « bezeichnet werden. Von den Vertragspartnern, also auch den NutzerInnen wird erwartet, dass sie das Qualitätsverständnis der Freien Wohlfahrtspflege (teilen). Diese Vorgaben lassen die Abkehr von einem institutionsorientierten Den- ken hin zu einem personorientierten Handeln zumindest fraglich erscheinen.

Aus professioneller Perspektive müssen die abzuschließenden Verträge - als wichtiges Qualitätskriterium - verfassungsund menschenrechtskonform sein. Da die Menschenrechte auf die Würde des Menschen bezogen sind, gelten auch sie selbst als »unveräußerlich ", wodurch sie sich prinzipiell von solchen Rechtsansprüchen unterscheiden, die durch politisch ausgehandelte, den Machtverhältnissen entsprechende Verträge oder gesetzgeberische Akte entstanden sind. (Bielefeldt 2004) Ein Vertrag wird also nicht nur im Hinblick auf seine Legalität (ist man ihm gegenüber gehorsam?), sondern auch seine Legitimität (ist er ethisch richtig oder zu kritisieren, zu verändern?) zu beurteilen sein (ausführlich dazu Thornhill/Ashenden 2010). So kann die Kürzung von Sozialhilfe unter das Existenzminimum oder gar auf Null - aus welchem Grund auch immer - aus philosophisch-menschenrechtlicher, aber auch aus bedürfnistheoretischer Sicht in keiner Weise legitimiert werden. Hartz IV ist leider ein Paradebeispiel blinder, legalistischer Gesetzesanwendung auf politisch dezisionistischer Grundlage, wobei in jüngster Zeit immer wieder neue Berechnungen aus dem Hut gezaubert werden. Wo bleiben die wissenschaftlichen Beiträge der Professionellen und Wohlfahrtsverbände zur bedürfnistheoretischen Begründung des notwendenden Bedarfs im Hinblick auf menschliches Wohlbefinden? Vielfach werden aber Bedürfnisse und Bedarf gleichgesetzt.

Grundanliegen: Ressourcenorientierung

Wohlfahrtsverbände fordern ein verantwortbares Umgehen mit humanen, finanziellen sowie ökologischen Ressourcen ... im Genaueren die Zusammenfübrung moderner betriebswirtschaftlicher Methoden mit gemeinwohlorientiertem Handeln,... .

"Zusammenführung « als Begriff lässt offen, wie die inhaltlich-theoretische Verhältnisbestimmung und Gewichtung zwischen Gemeinwohlorientierung und betriebswirtschaftlichem Management vorzunehmen ist. Insofern gibt es keine Möglichkeit, willkürliche Prioritätensetzungen aus professioneller Sicht zu kritisieren. Der Hinweis auf die europäisch anerkannten Normen (Din En ISO 9001:2000; EFQM-Modell für Excellen- 
ce) für das Qualitätsmanagement lässt eine Gewichtung zugunsten der Betriebswirtschaft vermuten. Dass auch die Profession Sozialer Arbeit verbindliche internationale Qualitätsstandards für Praxis und Studium entwickelt hat, kommt hier nicht in den Blick (vgl. Supplement 2007).

Wo bleiben die Qualitätsziele einer Profession Sozialer Arbeit?

Um auf diesem Hintergrund die Chancen einer Professionsentwicklung einschätzen zu können, müssten folgende Fragen beantwortet werden:

Haben die Sozialtätigen überhaupt ein Bewusstsein, Mitglied einer Profession zu sein, die sie verpflichtet, bei der Bestimmung von Qualitätszielen aufgrund ihres dritten wissenschafts- und menschenrechtsbezogenen Mandates mitzubestimmen? Wenn ja, können sie sich in den oberen Etagen entsprechend
Gehör verschaffen? Studien zeigen, dass sich die Zumutungen der neoliberalen Ideologie und die dazugehörigen Techniken/Tools - im Vergleich zu anderen Professionen - im Sozialwesen am ungebrochensten durchsetzen und etablieren konnten. (Exworthy 1999, Galuske/Thole 2006)

Sind SozialarbeiterInnen fähig, ihr Tun und Lassen auch wissenschaftlich und professionsethisch zu begründen und wie gehen sie mit den sich daraus ergebenden Widersprüchen und teilweise Unvereinbarkeiten zwischen Interessengruppen um?

Sind die Führungskräfte der Freien Wohlfahrtspflege bereit, ihre Qualitätsziele, ihr Menschen- und Gesellschaftsbild sowie die Vorstellungen über die Aufträge und Aufgaben Sozialer Arbeit sowie die teilweise widersprüchlichen Qualitätsziele einer »Revision « zu unterziehen?
Die Zukunft Sozialer Arbeit bleibt ungewiss. Professionalisierung bleibt ein unentschiedenes Projekt. Ich schließe dennoch mit einem richtungweisenden Zitat von Gore (1969):

"Die Menschenrechte ... werden diejenigen SozialarbeiterInnen verstören, die sich zur Ruhe gesetzt und mit den gerade herrschenden Werten und Theorien des lokalen Gemeinwesens (aber auch ihrer Träger/Arbeitgeber, StB) Frieden geschlossen haben - und zwar vor allem dann, wenn (sie) ... mit den Werten und Normen der Profession in Konflikt stehen. Menschenrechte werden von der organisierten Profession fordern, ja sie zwingen, zu sozialen (bzw. menschenrechtlichen) Fragen klar Stellung zu nehmen. Angesichts der Pluralität, die auch in der Sozialen Arbeit herrscht, sind die Menschenrechte ein notwendiger Maßstab und eine Orientierung für konstruktive Aktion. «(S. 68)

\title{
Vom Bundeskompetenzzentrums Barrierefreiheit e.V.
}

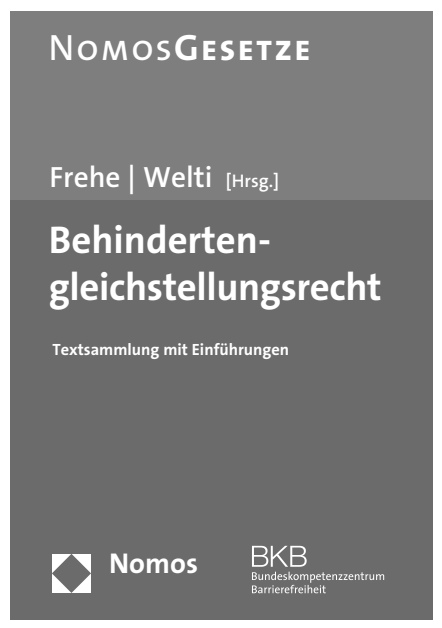

\author{
Behindertengleichstellungsrecht \\ Textsammlung mit Einführungen \\ Herausgegeben von Horst Frehe und Prof. Dr. Felix Welti \\ im Auftrag des Bundeskompetenzzentrums Barrierefreiheit e.V. (BKB) \\ 2010, 996 S., brosch., mit CD-ROM, 29,- €, ISBN 978-3-8329-5221-1
}

Seit einigen Jahren werden Behinderungen nicht mehr aus medizinischer Sicht definiert, sondern als Ergebnis einer Wechselwirkung zwischen individueller Beeinträchtigung und den Barrieren der Umwelt: Behindert ist man nicht, behindert wird man. Konsequenterweise verschiebt sich der Fokus von der Rehabilitation zur Herstellung von Chancengleichheit. Dieser Paradigmenwechsel spiegelt sich in einer Vielzahl von Normen auf allen Ebenen vom Völkerrecht bis zu den Rechtsvorschriften der deutschen Bundesländer. Allerdings sind die einschlägigen Bestimmungen nur schwer auffindbar, sodass die Betroffenen ihre Rechte und Pflichten nur mit großem Aufwand erfassen können.

In der vorliegenden Gesetzessammlung werden die Rechtsvorschriften zur Gleichstellung von Menschen mit Behinderung im Zusammenhang dargestellt. Die Sammlung ist systematisch in fünf Abschnitte zum Völkerrecht, dem Europäischen Recht, dem Verfassungsrecht des Bundes und der Länder, dem Verwaltungsrecht des Bundes und der Länder sowie den einschlägigen Bestimmungen des Zivilrechts gegliedert. Den einzelnen Abschnitten und Unterabschnitten ist jeweils eine Einleitung vorangestellt, aus der sich die Bedeutung der aufgeführten Normen erschließt.

Bitte bestellen Sie im Buchhandel oder

versandkostenfrei unter $\checkmark$ www.nomos-shop.de 